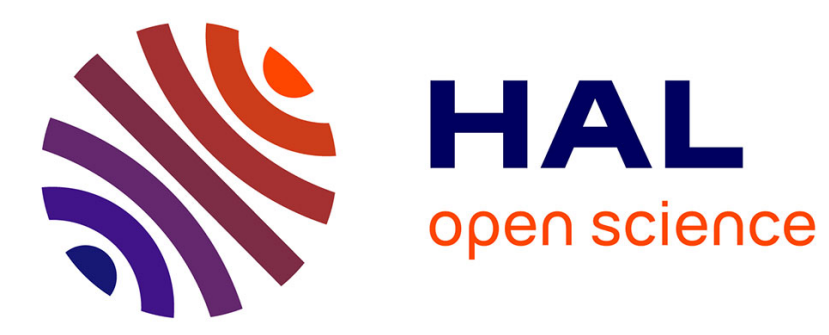

\title{
Problèmes méthodologiques posés par l'extraction et la récupération des bactéries telluriques pour leur quantification par immunofluorescence \\ Yves Crozat, Jean-Claude Cleyet-Marel
}

\section{- To cite this version:}

Yves Crozat, Jean-Claude Cleyet-Marel. Problèmes méthodologiques posés par l'extraction et la récupération des bactéries telluriques pour leur quantification par immunofluorescence. Agronomie, 1984, 4 (7), pp.603-610. hal-00884678

\section{HAL Id: hal-00884678 \\ https://hal.science/hal-00884678}

Submitted on 1 Jan 1984

HAL is a multi-disciplinary open access archive for the deposit and dissemination of scientific research documents, whether they are published or not. The documents may come from teaching and research institutions in France or abroad, or from public or private research centers.
L'archive ouverte pluridisciplinaire HAL, est destinée au dépôt et à la diffusion de documents scientifiques de niveau recherche, publiés ou non, émanant des établissements d'enseignement et de recherche français ou étrangers, des laboratoires publics ou privés. 


\title{
Problèmes méthodologiques posés par l'extraction et la récupération des bactéries telluriques pour leur quantifi- cation par immunofluorescence
}

Yves CROZAT \& Jean-Claude CLEYET-MAREL (*)

E.S.A., 24, rue Auguste-Fonteneau, F 49004 Angers

(*) I.N.R.A., Laboratoire de Recherches sur les Symbiotes des racines,

9, place Viala, F 34060 Montpellier Cedex.

\begin{abstract}
Afin d'améliorer la valeur quantitative de la technique de dénombrement des bactéries telluriques (Rhizobium japonicum) par immunofluorescence, on compare différents modes d'extraction et de récupération.

L'utilisation de membranes en polycarbonates (Nucléopores) supprime les 40 p. 100 de pertes occasionnées par les membranes cellulosiques (Millipores) classiquement employées. Parmi les différents floculants testés, $\mathrm{Ca}(\mathrm{OH})_{2} / \mathrm{MgCO}_{3}(2 / 5)$ permet d'obtenir les meilleurs résultats. Cependant pour les deux sols tropicaux étudiés, une floculation par centrifugation est nécessaire.

Lorsque l'étape de la floculation n'est pas limitante, la dispersion du sol dans un extractif composé de gélatine diluée dans une solution de $\left(\mathrm{NH}_{4}\right)_{2} \mathrm{HPO}_{4} 0,1 \mathrm{M}$ améliore significativement le taux de récupération des bactéries.
\end{abstract}

Mots clés additionnels : Rhizobium japonicum, écologie microbienne.

We have tested different procedures for releasing bacteria (Rhizobium japonicum) from soil particles and collecting them for fluorescent antibody quantification. The use of polycarbonate filters (Nucleopore) avoided the $40 \%$ loss of bacteria usually found with classical cellulose filters (Millipore).

A flocculant mixture of $\mathrm{Ca}(\mathrm{OH})_{2} / \mathrm{MgCO}_{3}(2 / 5)$ gave the best results. However flocculation by centrifugation was needed for the two tropical soils tested. When the flocculation step was not the limiting factor, partially hydrolysed gelatin diluted in ammonium phosphate $(0.1 \mathrm{M})$ could be used to suspend the soil and increase the recovery.

Additional key words : Rhizobium japonicum, microbial ecology.

\section{INTRODUCTION}

Les techniques de marquage par immunofluorescence (IF) sont des outils très efficaces d'identification et de dénombrement des micro-organismes dans leur milieu naturel (BOHLOOL \& SCHMIDT, 1980).

La technique de dénombrement sur membrane de filtration décrite par SCHMIDT (1974) permet de quantifier spécifiquement des populations bactériennes dans le sol et la rhizosphère (CLEYET-MAREL \& CROZAT, 1982 ; CROZAT et al., 1982). Une des originalités de cette technique consiste à séparer les particules minérales et organiques des bactéries et à concentrer ces der- nières sur une membrane de filtration selon un protocole dont les principales étapes sont les suivantes :

- dispersion de $10 \mathrm{~g}$ de sol dans $95 \mathrm{ml} \mathrm{d'eau} \mathrm{distil-}$ lée, à l'aide d'un malaxeur type Waring-blendor (EBERBACK corporation) afin d'extraire les bactéries des particules de sol ;

- floculation des colloïdes à l'aide de $0,7 \mathrm{~g}$ d'un mélange de $\mathrm{Ca}(\mathrm{OH})_{2} / \mathrm{MgCO}_{3}(2 / 5)$ pour obtenir un surnageant limpide contenant un maximum de bactéries ;

- après $10 \mathrm{mn}$, récupération d'un aliquote du surnageant et piégeage des bactéries par filtration sur une membrane cellulosique (Millipore Sa. 0,45 $\mu \mathrm{m}-20 \mathrm{~mm}$ de diamètre) ; 
- marquage des bactéries à l'aide d'anticorps spécifiques conjugués à l'isothiocyanate de fluorescéine. Observation au microscope par épi-fluorescence. Le nombre de bactéries/g de sol est estimé par la formule suivante :

$$
y=\bar{x} \cdot \frac{S}{s} \cdot \frac{d}{v}
$$

y : nombre de bactéries/g de sol

$\overline{\mathrm{X}}$ : nombre moyen de bactéries/champ de microscope

$\mathrm{S}$ : surface effective de filtration

$s$ : surface d'un champ de microscope

$\mathrm{d}$ : facteur de dilution (10 généralement)

$\mathrm{v}$ : volume de surnageant filtré ( $10 \mathrm{ml}$ au maximum).

Les 3 premières étapes ne permettent pas une récupération totale de bactéries. Le taux de récupération est une caractéristique du sol et de l'espèce bactérienne considérée. REYES \& SCHMIDT (1979) obtiennent avec un sol de Waukegan aux Etats-Unis, un taux de récupération de 35 p. 100 avec la souche 123 de $R$. japonicum, alors que KINGSLEY \& BOHLOOL (1981) rapportent des taux inférieurs à 1 p. 100 dans des sols tropicaux avec la même souche. D'autres auteurs (WOLLUM \& Miller, 1980 ; BezDiCEK \& DONALDSON, 1980) ont, dans le cas de fortes populations microbiennes, des rendements de 100 p. 100 grâce à une centrifugation du surnageant dans un gradient de saccharose. Plus récemment, KInGSLEY \& BoHLOOL (1981) ont réussi à faire passer le taux de 1 p. 100 de certains sols tropicaux à près de $100 \mathrm{p} .100$ en modifiant les conditions d'extraction avant floculation.

Nous nous proposons ici de comparer différentes procédures d'extraction et de récupération des bactéries telluriques, afin d'améliorer la valeur quantitative de la technique de dénombrement par immunofluorescence dans le cadre d'études autoécologiques et de l'adapter à une plus grande variété de sols.

\section{MATÉRIEL ET MÉTHODES}

\section{A. Dénombrement des suspensions bactériennes}

La souche de $R$. japonicum $\mathrm{G}_{2} \mathrm{sp}$, mutant spontané résistant à la spectinomycine de la souche $\mathrm{G}_{2}$ (311 B135 USDA Beltsville) est cultivée sur milieu solide à base d'extrait de levure et de mannitol (VINCENT, 1970). Après 4 ou $5 \mathrm{j}$ de culture à $28^{\circ} \mathrm{C}$, les bactéries sont reprises dans $9 \mathrm{ml}$ d'eau stérile. Cette suspension est diluée de 10 en 10 et $1 \mathrm{ml}$ des suspensions bactériennes, proches de $10^{5}-10^{6}$ bactéries $/ \mathrm{ml}$, est dilué dans $1 \mathrm{ml}$ d'une solution d'acide picrique à $1 \mathrm{p}$. 100 . On fait 10 dépôts de $25 \mu \mathrm{l}$ de cette nouvelle suspension sur les empreintes circulaires $(\varnothing 7 \mathrm{~mm}$ ) d'une lame microprint. La préparation est fixée à la chaleur et les bactéries sont marquées avec un conjugué fluorescent spécifique avant d'être observées. Ces dénombrements sur lame, utilisés comme référence, seront ensuite comparés aux dénombrements sur membranes de filtration.

\section{B. Comparaison de différents types de membranes de filtration}

Deux types de membranes sont testés : des membranes cellulosiques (type Millipore Sa. 0,45 $\mu \mathrm{m}$ ) et des membranes en polycarbonates (type Nucléopore corp. $-0,40 \mu \mathrm{m})$.

\section{Membranes cellulosiques}

La souche $\mathrm{G}_{2}$ sp est cultivée et reprise comme précédemment, pour chaque dilution, 3 aliquotes d'un volume, variable suivant le rang de dilution, sont filtrés sur une membrane préalablement marquée à l'encre de chine (PAILLARD, SA). Les membranes sont ensuite traitées pour un dénombrement en immunofluorescence selon la technique décrite par SCHMIDT (1974).

\section{Membranes en polycarbonates}

Le protocole est identique à celui suivi pour les membranes cellulosiques mais, avant utilisation, elles sont traitées de la façon suivante :

Les filtres sont immergés, sous vide partiel, dans une solution d'A.C.S. (Acide cholique sel de Sodium) à 0,5 p. 100 pendant $5 \mathrm{mn}$, puis $22 \mathrm{~h}$ dans du Noir d'Irgalan (Ciba-Geigy, Irgalan black, BGL 200) à 0,2 p. 100 dans une solution d'acide citrique à 2 p. 100 .

Les membranes sont ensuite séchées à $90^{\circ} \mathrm{C}$ pendant $1 \mathrm{~h}$ et rincées dans de l'eau déminéralisée avant d'être stockées sous atmosphère sèche.

\section{Etude de différents modes de floculation}

Différents modes de floculation sont testés pour 4 sols agricoles : 2 de région tempérée et 2 de régions tropicales (tabl. 1). La limpidité du surnageant est estimée par densité optique à $660 \mathrm{~nm}$.

\section{Sols de régions tempérées (Lyon-Lavalette)}

On étudie l'évolution de la limpidité du surnageant obtenue avec 3 floculants chimiques :

$$
\begin{aligned}
& -0,9 \text { et } 0,7 \mathrm{~g} \text { de } \mathrm{Ca}(\mathrm{OH})_{2} / \mathrm{MgCO}_{3}(2 / 5) \\
& -1 \mathrm{ml} \mathrm{de} \mathrm{CaCl}_{2} \text { à } 1 \mathrm{p} .100 \\
& -1 \mathrm{ml} \mathrm{d} \mathrm{AlCl}_{3} \text { à } 0,1 \mathrm{p} .100 .
\end{aligned}
$$

On estime également, pour le sol de Lavalette, la perte de bactéries occasionnée par la floculation au cours du temps.

\section{Sols de régions tropicales (Rwanda-Madagascar)}

Ces sols ne présentent aucune floculation significative par voie chimique. On étudie un mode de clarification du surnageant $(10 \mathrm{ml})$ par centrifugation à différentes vitesses durant $5 \mathrm{mn}$. Deux modes d'extraction des bactéries sont utilisés : de l'eau distillée ou de la gélatine à 1 p. 100 hydrolysée, diluée dans $\left(\mathrm{NH}_{4}\right)_{2}$ $\mathrm{HPO}_{4} 0,1 \mathrm{M}$. Les pertes de bactéries lors des centrifugations sont estimées. 
TABLEAU 1

Propriétés physico-chimiques des sols de régions tempérées (Lyon-Lavalette) et tropicales (Madagascar-Rwanda). Physical and chemical properties of temperate (Lyon-Lavalette) and tropical soils (Madagascar-Rwanda).

\begin{tabular}{|c|c|c|c|c|}
\hline & Lyon & Lavalette & Madagascar & Rwanda \\
\hline \multicolumn{5}{|l|}{ Granulométrie en $\%$} \\
\hline Sables grossiers : $0,2-2 \mathrm{~mm}$ & 167 & 159 & - & 246 \\
\hline Sables fins $\quad: 0,050-0,2 \mathrm{~mm}$ & 155 & 171 & - & 146 \\
\hline Limons grossiers : $0,020-0,050 \mathrm{~mm}$ & 113 & 154 & - & 136 \\
\hline Limons fins $\quad: 0,002-0,020 \mathrm{~mm}$ & 251 & 342 & - & 164 \\
\hline Argiles $\quad: 0-0,002 \mathrm{~mm}$ & 314 & 174 & - & 308 \\
\hline Carbone organique (méthode ANNE) $\%$ & 6,1 & 7,2 & 4,07 & 4,61 \\
\hline$\cdot \mathrm{C} / \mathrm{N}$ & 7,6 & 10 & & 10,5 \\
\hline \multirow[t]{3}{*}{ - $\mathrm{pH}$ eau } & 6,6 & 8,2 & 4,86 & 4,1 \\
\hline & & & (ferralitique & $\mathrm{Al} / \mathrm{meq} / 100 \mathrm{~g}$ \\
\hline & & & $\begin{array}{l}\text { fortement } \\
\text { désaturé) }\end{array}$ & \\
\hline
\end{tabular}

\section{Etude et amélioration du taux de récupération des bactéries à partir de différents sols}

Une solution de gélatine (DIFCO) à 1 p. 100 est préparée dans de l'eau distillée. Après ajustement à pH 10,3 et hydrolyse partielle de $10 \mathrm{mn}$ à $121^{\circ} \mathrm{C}$, cette solution est diluée au $1 / 10$ dans $\left(\mathrm{NH}_{4}\right)_{2} \mathrm{HPO}_{4} 0,1 \mathrm{M}$. De cette solution finale, $100 \mathrm{ml}$ servent de dispersant et d'extractif lors de la $1^{\text {re }}$ étape. Mis à part le temps de floculation qui doit être prolongé de $30 \mathrm{mn}$ en raison de la turbidité du milieu, les étapes suivantes sont identiques à la méthode décrite par SCHMIDT (1974). Les taux de récupération des bactéries de 2 sols de régions tempérées (Lyon-Lavalette) et de 2 sols de régions tropicales (Rwanda et Madagascar) sont étudiés (tabl. 2).

1) Par la méthode classique : dispersion et extrac- tion des bactéries dans $100 \mathrm{ml}$ d'eau distillée, filtration du surnageant sur membranes cellulosiques après floculation.

2) Par la méthode classique mais en remplaçant les membranes en acétate de cellulose par des membranes en polycarbonate.

3) Par dispersion et extraction des bactéries dans $100 \mathrm{ml} \mathrm{du}$ dispersant décrit ci-dessus et filtration du surnageant après floculation sur membranes en polycarbonates.

Les différents protocoles sont mis en œuvre $16 \mathrm{~h}$ après inoculation $(2 \mathrm{ml}$ de suspension de la souche $\mathrm{G}_{2} \mathrm{sp}$ pour $10 \mathrm{~g}$ de sol).

Le contrôle du nombre de bactéries introduites dans les sols par inoculation est réalisé sur lames microprint. Le taux de récupération est alors apprécié à l'aide du rapport :

$$
\mathbf{R}=\frac{\text { nombre de bactéries par } \mathbf{g} \text { de sol dénombrées sur membrane }}{\text { nombre de bactéries inoculées par } \mathbf{g} \text { de sol }}
$$

TABLEAU 2

Estimation du taux de récupération de dénombrement sur membrane de filtration de populations de $\mathrm{R}$. japonicum (souche $G_{2}$ sp) introduites dans des sols tempérés et dans des sols tropicaux.

Recovery rate estimations of filter counts of $\mathrm{R}$. japonicum (strain $G_{2} s p$ ) populations introduced in temperate soils and in tropical soils.

\begin{tabular}{lccc}
\hline \multicolumn{1}{c}{ Protocole } & Sols de zone tempérée & \multicolumn{2}{c}{ Sols de zone tropicale } \\
\cline { 2 - 4 } & Lavalette & Lyon & Madagascar \\
\hline $\begin{array}{l}\text { Classique : eau + membrane cellulosique (ScHMid, 1974) } \\
\text { (a) }\end{array}$ & $13\left(^{*}\right)$ & 22,5 & - \\
\hline $\begin{array}{l}\text { Eau + membrane en polycarbonates } \\
\text { (b) }\end{array}$ & 24,5 & 27,5 & 4,6 \\
\hline $\begin{array}{l}\left.\text { Dispersion du sol dans un extractif : « gélatine-( } \mathrm{NH}_{4}\right)_{2} \mathrm{HPO}_{4} 》+\text { membrane } \\
\text { en polycarbonates } \\
\text { (c) }\end{array}$ & 73 & 37 & 11,5 \\
\hline
\end{tabular}

$\left(^{*}\right.$ Taux de récupération exprimé en $\%$. 


\section{RÉSULTATS}

\section{A. Comparaison de différents types de membranes de filtration}

La comparaison des dénombrements obtenus sur membrane après filtration d'une suspension bactérienne avec les dénombrements de référence sur lame est représentée figure 1, pour les membranes cellulosiques, et figure 2 , pour les membranes en polycarbonates. Dans les 2 cas, l'ensemble des points s'ajustent à

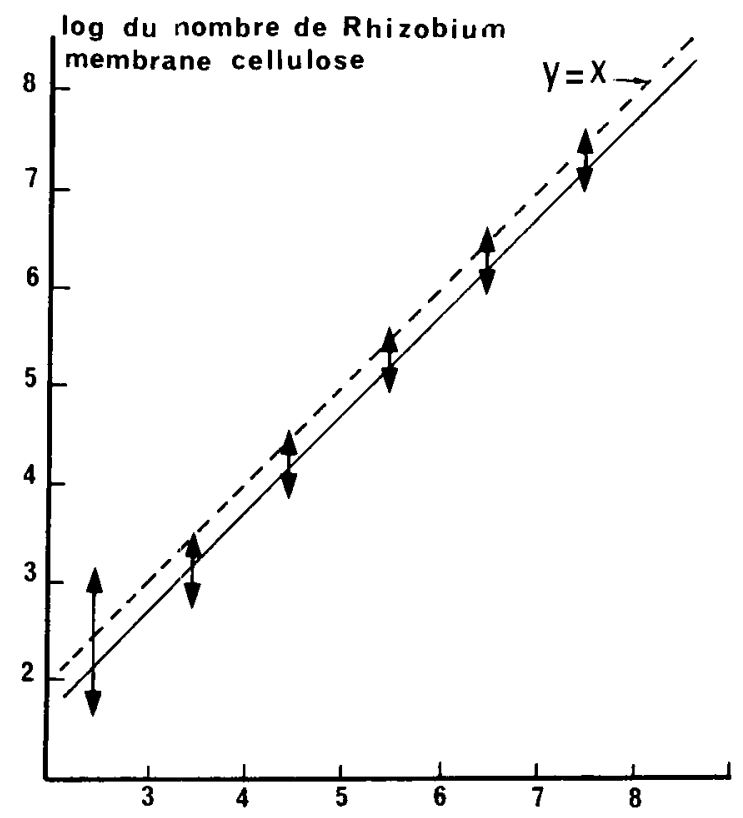

log du nombre de Rhizobium; sur lames

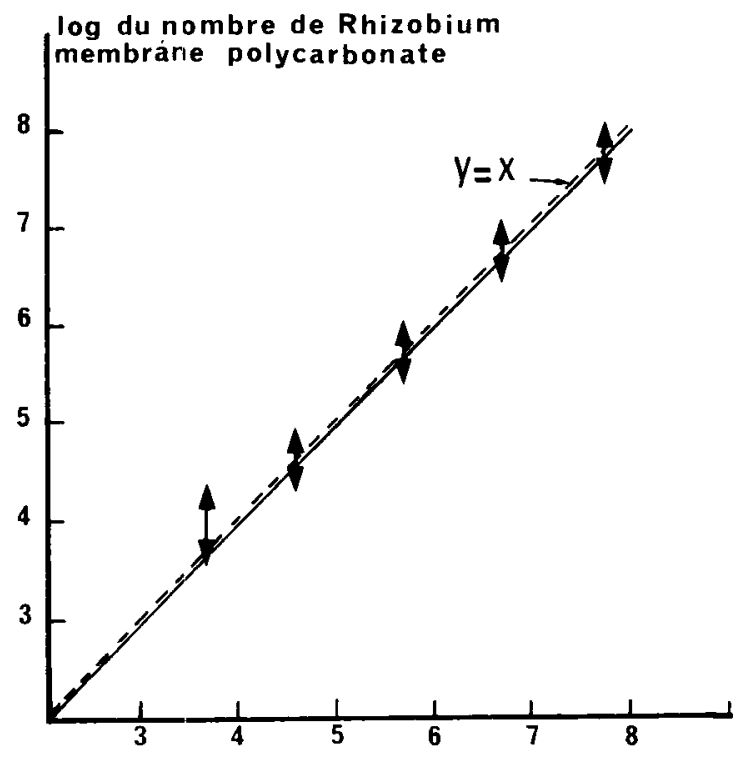

log du nombre de Rhizobium; sur lames

Figures 1 et 2 .

Cinétique de récupération de $\mathrm{R}$. japonicum (souche $G_{2} s p$ ) sur membranes cellulosiques (I) et sur membranes en polycarbonates (2) en fonction de la population présente dans la suspension avant filtration.

Kinetics of recovery of $\mathrm{R}$. japonicum (strain $G_{2}$ sp) on cellulose filters (1) and on polycarbonate filters (2) plotted against the population in the suspension before filtration. des fonctions linéaires. Ces résultats montrent la bonne reproductibilité de la méthode de dénombrement pour des conditions opératoires constantes. En dessous de $10^{4}$ bactéries $/ \mathrm{ml}$, la précision des dénombrements s'avère plus faible compte-tenu des intervalles de confiance définis par la loi de Porsson. En déterminant la pente des différentes droites obtenues en nombre réel, on peut estimer les taux de récupération de chacune des membranes : soit pour les membranes cellulosiques, une constante de 0,603 et, pour les membranes en polycarbonate, une constante de 0,975 . Ces dernières autorisent donc une récupération proche de $100 \mathrm{p} .100$, alors que celle de la méthode de référence n'est que de 60 p. 100. De plus, il faut noter que la qualité de l'observation est nettement améliorée avec ce type de membrane: les bactéries se trouvent toutes sur un même plan d'observation; il n'en est pas de même avec les membranes cellulosiques.

\section{B. Etude de différents modes de floculation}

\section{Sols de régions tempérées (Lyon-Lavalette)}

L'évolution des valeurs de densité optique à $660 \mathrm{~nm}$ obtenues avec les différents floculants est donnée pour chacun des sols (fig. 3). Le mélange floculant constitué

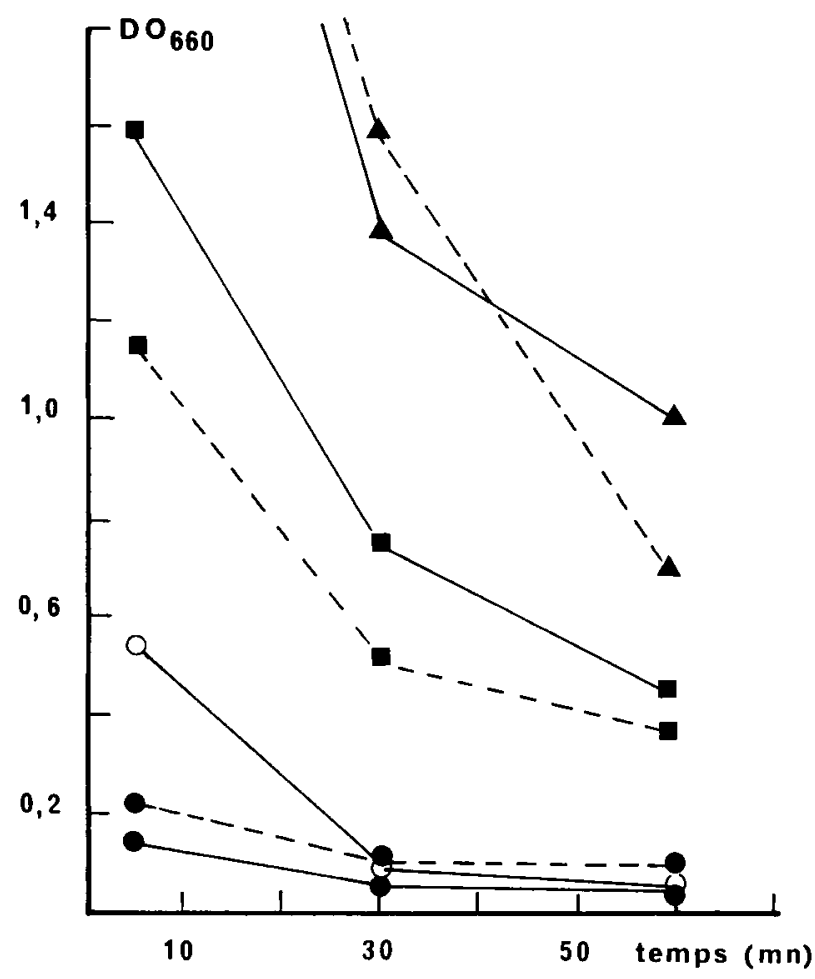

Figure 3 .

Evolution de la densité optique du surnageant avec différents agents floculants pour les sols de régions tempérées.

$$
\begin{aligned}
& \text { Sol de Lyon :----- } \square \mathrm{CaCl}_{2} \\
& \text { Sol de Lavalette : __ } \mathrm{Ca}(\mathrm{OH})_{2}-\mathrm{MgCO}_{3}=0,7 \mathrm{~g} \\
& \mathrm{Ca}(\mathrm{OH})_{2}-\mathrm{MgCO}_{3}=0,2 \mathrm{~g} \\
& \triangle \mathrm{AlCl}_{3} \text {. }
\end{aligned}
$$

Change in the optical density of the supernatant with different flocculants for temperate soils.

Lyon soil : - ---

$$
\mathrm{CaCl}_{2}
$$

Lavalette soil :

$$
\begin{aligned}
& \mathrm{Ca}(\mathrm{OH})_{2}-\mathrm{MgCO}_{3}=0.7 \mathrm{~g} \\
& \mathrm{Ca} \mathrm{OH})_{2}-\mathrm{MgCO}_{3}=0.2 \mathrm{~g} \\
& 4 \mathrm{AlCl}_{3} .
\end{aligned}
$$


de $\mathrm{Ca}\left(\mathrm{OH}_{2}\right)$ et de $\mathrm{MgCO}_{3}(2 / 5)$ à raison de $0,7 \mathrm{~g}$ pour $10 \mathrm{~g}$ de sol se révèle le plus efficace. Il permet d'obtenir un surnageant très clair $(\mathrm{D} . \mathrm{O} .=0,2)$ en un minimum de temps. Plus le temps de floculation est long, plus le risque de pertes de bactéries par gravité augmente (fig. 4). Pour le sol de Lavalette ces pertes sont minimum pour des temps de floculation de $10 \mathrm{mn}$.

Quant aux autres types de floculants, pour ces sols, même après un temps de floculation de $60 \mathrm{mn}$, ils ne permettent pas une bonne précipitation des particules. Les densités optiques obtenues restent trop élevées (D.O. $>0,2)$ pour de bonnes observations sur les membranes de filtration.

\section{Sols de régions tropicales (Rwanda-Madagascar)}

La centrifugation de $10 \mathrm{ml}$ de surnageant pour des vitesses supérieures ou égales à 2000 tours. $\mathrm{mn}^{-1}$, pendant $5 \mathrm{mn}$ permet de récupérer un surnageant ayant une D.O. inférieure à 0,3 (fig. 5 ). Des vitesses de centrifugation plus élevées modifient relativement peu la limpidité du surnageant quel que soit le soluté dans lequel les bactéries sont dispersées (fig. 6). En raison des difficultés d'observation dues aux particules en suspension dans le surnageant, le meilleur compromis consiste à centrifuger celui-ci pendant $5 \mathrm{mn}$ à 2000 tours. $\mathrm{mn}^{-1}$. La perte relative de bactéries est alors de 27 p. 100.

\section{Etude et amélioration du taux de récupération des différents sols}

Les qualités d'extraction et de récupération des différents protocoles expérimentaux testés pour les 4 sols étudiés sont présentés dans les figures 7A, B, C, D.

La droite $\mathrm{y}=\mathrm{x}$ tracée sur chacune des figures correspond à un taux de récupération maximum de 100 p. 100. Dans ce cas, on recenserait autant de bactéries après traitement des échantillons de sol (y) qu'il y en a d'inoculées dans l'échantillon (x). Plus la courbe expérimentale de récupération est proche de cette $1^{\text {re }}$ bissectrice et plus le protocole utilisé est performant. Les moins bonnes récupérations sont obtenues avec le protocole classique (SCHMIDT, 1974) et la supériorité des membranes en polycarbonate se trouve confirmée par ces résultats.

La dispersion du sol dans $95 \mathrm{ml}$ d'un extractif composé de gélatine (à 1 p. 100) partiellement hydrolysée et diluée au $1 / 10$ dans une solution de $\left(\mathrm{NH}_{4}\right) \mathrm{HPO}_{4}$ $0,1 \mathrm{M}$ couplée à l'utilisation de membranes en polycarbonates améliore les cinétiques de récupération dans tous les sols.

Pour des densités microbiennes inférieures à des densités de l'ordre de $10^{4}$ Rhizobium/g de sol la récupération des bactéries est moins que proportionnelle (seuil de non-proportionnalité). Il y a alors des risques de surestimation de la population microbienne. Pour certains protocoles, avec de très faibles populations $\left(10^{2}, 10^{3}\right.$ Rhizobium/g de sol), le taux de récupération dépasse $100 \mathrm{p} .100$. A ces niveaux de population, le nombre de bactéries par champ de microscope est faible $(<$ à 0,3$)$ et il est alors nécessaire de filtrer un maximum de surnageant $(10 \mathrm{ml})$ pour le compenser. Il s'ensuit une augmentation du nombre de particules

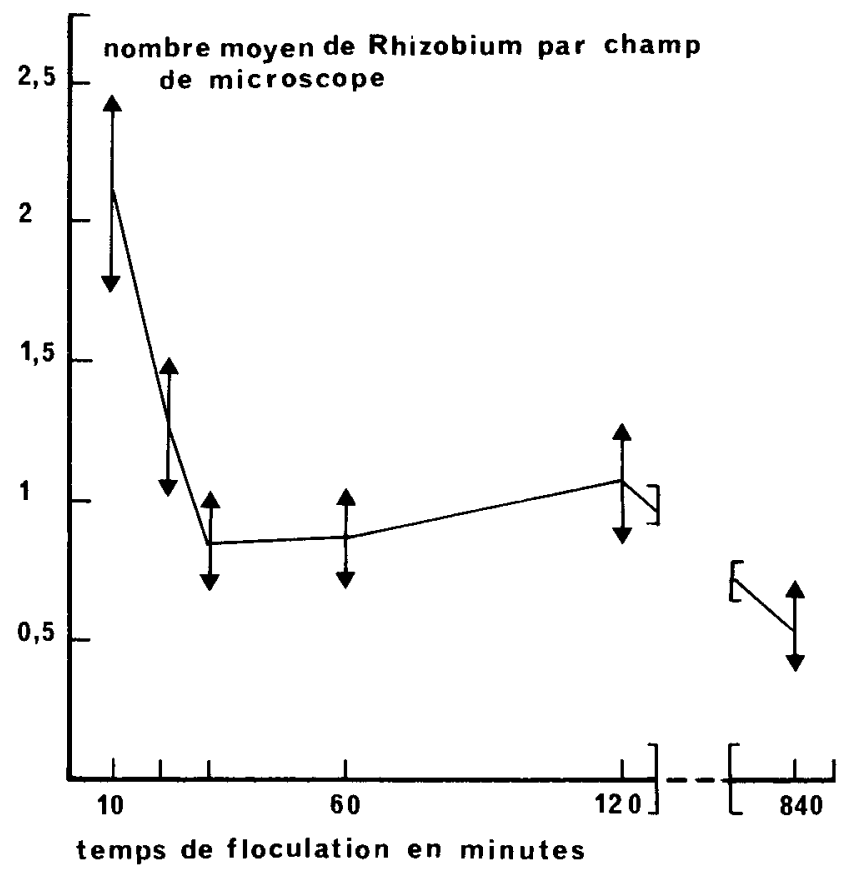

Figure 4.

Effet du temps de floculation sur le nombre de Rhizobium (souche $G_{2} s p$ ) récupérés sur membrane de filtration en polycarbonates (sol de région tempérée : Lavalette).

Effect of time of flocculation on the number of Rhizobium (strain $\left.G_{2} s p\right)$ trapped on the filter (temperate soil : Lavalette).

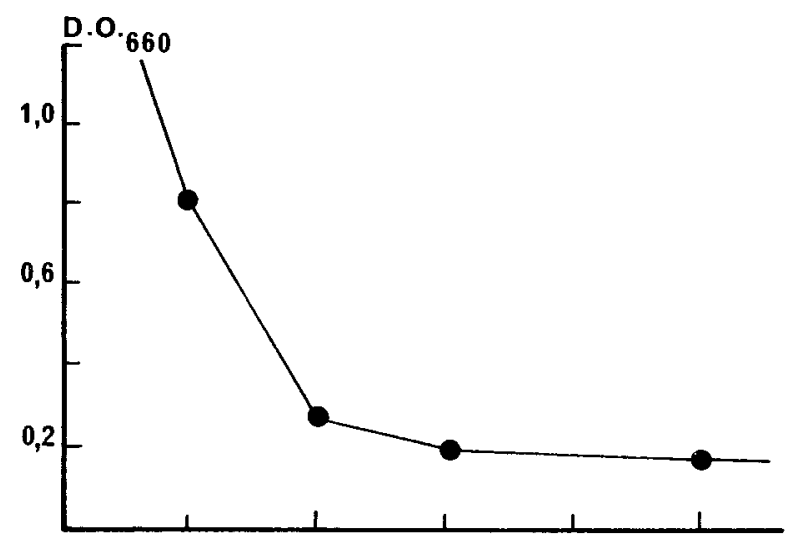

Vitesse de centrifugation. tours/minute

Figure 5 .

Effet de la centrifugation sur la densité optique du surnageant. Sols tropicaux.

Effect of centrifugation on the optical density of the supernatant. Tropical soils.

minérales et organiques qui peuvent être la source d'artefacts dont l'incidence n'est plus négligeable. Sur les graphiques, on observe que le seuil de nonproportionnalité est d'autant plus élevé que la qualité de récupération du protocole utilisé est plus faible. Pour des populations supérieures à ce seuil, il est possible d'ajuster les différentes cinétiques à des droites. On peut alors estimer une constante de récupération pour chacun des sols avec les différents protocoles testés (tabl. 2). 


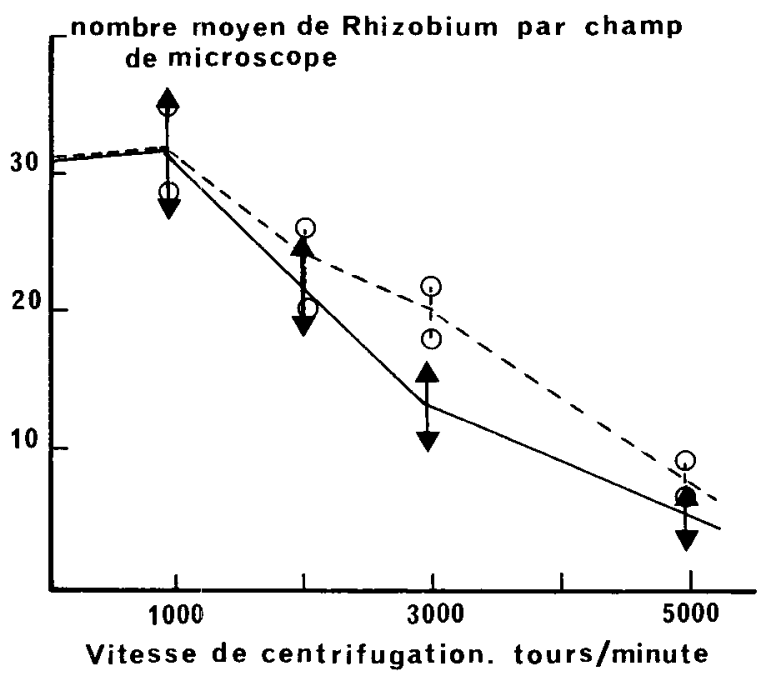

Figure 6.

Effet de la centrifugation d'une suspension de Rhizobium (souche $\left.G_{2} s p\right)$ sur le nombre de bactéries récupérées sur membrane de filtration en polycarbonate.

(ـ dilution et centrifugation dans un extractif de gélatine hydrolysée, diluée dans une solution de $\left(\mathrm{NH}_{4} \mathrm{~J}_{2} \mathrm{HPO}_{4} \mathrm{O}, \mathrm{I} \mathrm{M}\right.$. (----) dilution et centrifugation dans $\mathrm{H}_{2} \mathrm{O}$.

Effect of centrifugation of a Rhizobium suspension (strain $G_{2} s p$ ) on the number of bacteria trapped on the filter.

( $\longrightarrow$ dilution and centrifugation in an extractant composed of hydrolysed gelatine diluted in $\left(\mathrm{NH}_{4}\right)_{2} \mathrm{HPO}_{4} 0.1 \mathrm{M}$.

(-----) dilution and centrifugation in $\mathrm{H}_{2} \mathrm{O}$.

\section{DISCUSSION}

Lorsqu'on observe directement une goutte de suspension de sol ou une lame après enfouissement dans le sol, il faut environ $10^{7}$ bactéries $/ g$ de sol pour compter, par immunofluorescence, au moins une cellule par champ de microscope (SCHMIDT, 1974). Par extraction et concentration des bactéries sur une membrane de filtration, il est devenu possible de quantifier avec fiabilité des populations de l'ordre de $10^{4}$ bactéries $/ \mathrm{g}$ de sol (SCHMIDT, 1978). Le seuil théorique d'utilisation de cette méthode dépend du matériel utilisé (microscope, diamètre des membranes de filtration), de la précision désirée et du temps consacré à l'observation.

Généralement, le rapport $\mathrm{S} / \mathrm{s}$ (Surface de filtration/ surface du champ du microscope) est de l'ordre de $10^{4}$ $\left(1,56.10^{4}\right.$ avec notre matériel) et, $\frac{\mathrm{d}}{\mathrm{v}}$ (facteur de dilution/volume du surnageant) est au minimum égal à 1 . Nous savons que le recensement de 100 individus sur une membrane permet d'estimer la population bactérienne avec une incertitude relative de 22 p. 100 alors que, pour 50 individus, elle est de 30 p. 100 (CLEYETMAREL \& CHESSEL, 1978). Dans ces conditions, pour conserver une incertitude relative de $30 \mathrm{p} .100$, il faudra observer 50 champs de microscope si la population bactérienne est de $1,56.10^{4} / \mathrm{g}$ de sol et 500 si elle est de $1,56.10^{3} / \mathrm{g}$ de sol.

Sachant qu'il faut environ $25 \mathrm{mn}$ pour observer 100 champs et qu'en cas d'observation prolongée le risque d'erreur d'identification s'accroît, il est difficile de dépasser 150 champs d'observations par membrane de filtration. Pour des populations microbiennes inférieures à $5.10^{3}$ bactéries/g de sol, les dénombrements seront entachés d'une incertitude relative supérieure à 30 p. 100. Cependant, dans la réalité, ce seuil d'utilisation est souvent supérieur dans la mesure où il est rare de récupérer 100 p. 100 des bactéries. Par exemple, les taux de 10 p. 100 (cas des sols des régions tropicales étudiés) déterminent un seuil d'utilisation de $5.10^{4}$ pour 150 champs observés si on se fixe une incertitude relative de 30 p. 100. Dans le cas d'études d'autoécologie, un taux de récupération maximum permet donc d'accroître les possibilités d'étude en milieu réel en améliorant la précision des mesures. L'utilisation de membranes en polycarbonates préalablement traitées à l'ACS et au noir d'Irgalan permet de s'affranchir des 40 p. 100 de pertes liées à la structure (réseau de fibrilles d'une épaisseur de $120 \mu \mathrm{m}$ ) des membranes cellulosiques tout en conservant une répartition des bactéries distribuée selon une loi de POISSON (CROZAT, 1983).

L'extraction des bactéries dans de l'eau lors de la dispersion du sol par un Waring-blendor n'est pas totale. Elle est améliorée par l'utilisation de la gélatine hydrolysée, diluée dáns une solution de phosphate d'ammonium. Ceci est conforme aux résultats de KINGSLEY \& BOHLOOL (1981).

Cette amélioration, très variable selon les sols considérés, est plus élevée pour des sols présentant une bonne floculation (sols des régions tempérées) et beaucoup plus faible pour des sols floculants difficilement (sols des régions tropicales).

Malgré la présence d'oxydes métalliques qui rendent plus difficile l'extraction des bactéries des colloïdes, KINGSLEY \& BOHLOOL (1981), à l'aide du même extractif, font pourtant passer le taux de récupération de différents sols tropicaux de 1 p. 100 (dispersion dans l'eau distillée) à plus de $90 \mathrm{p}$. 100. Ceci laisse à penser que, dans notre cas, la floculation est le facteur le plus limitant du taux de récupération.

Certains auteurs (BEZDICEK \& DONALDSON, 1980) montrent que, pour différents sols, le mélange de $\mathrm{Ca}(\mathrm{OH})_{2} / \mathrm{MgCO}_{3}$ est le floculant qui engendre le plus de pertes de bactéries. $\mathrm{CaCl}_{2}$ et $\mathrm{AlCl}_{3}$ donnent de meilleurs taux de récupération, mais avec des surnageants moins limpides.

$\mathrm{Si}$, dans le cas de fortes densités microbiennes ( $>10^{6} / \mathrm{g}$ de sol), une faible limpidité est sans problème en raison des très faibles quantités de surnageants filtrées $(\simeq 1 \mathrm{ml})$, elle est par contre très problématique pour des populations plus faibles. C'est pourquoi l'utilisation du mélange $\mathrm{Ca}(\mathrm{OH})_{2} / \mathrm{MgCO}_{3}$ est, malgré tout, le meilleur compromis. En ce qui concerne les sols tropicaux, d'autres floculants chimiques sont à l'étude.

D'une manière générale, toutes les cinétiques de récupération montrent que, dans le cas de faibles populations bactériennes inférieures au seuil réel d'utilisation (seuil théorique $\times$ taux de récupération), les dénombrements sont sujets à caution. Les erreurs d'identification sont plus importantes et des variations de population ne sont pratiquement pas mises en évidence (fig. 7E).

$\mathrm{Au}$-delà du seuil de résolution, la récupération peut être considérée comme linéaire. La valeur du seuil de résolution dépend du sol utilisé et il est d'autant plus bas que le taux de récupération est plus élevé. 
log du nombre de Rhizobium dénombres par gramme de sol par immunofluorescence
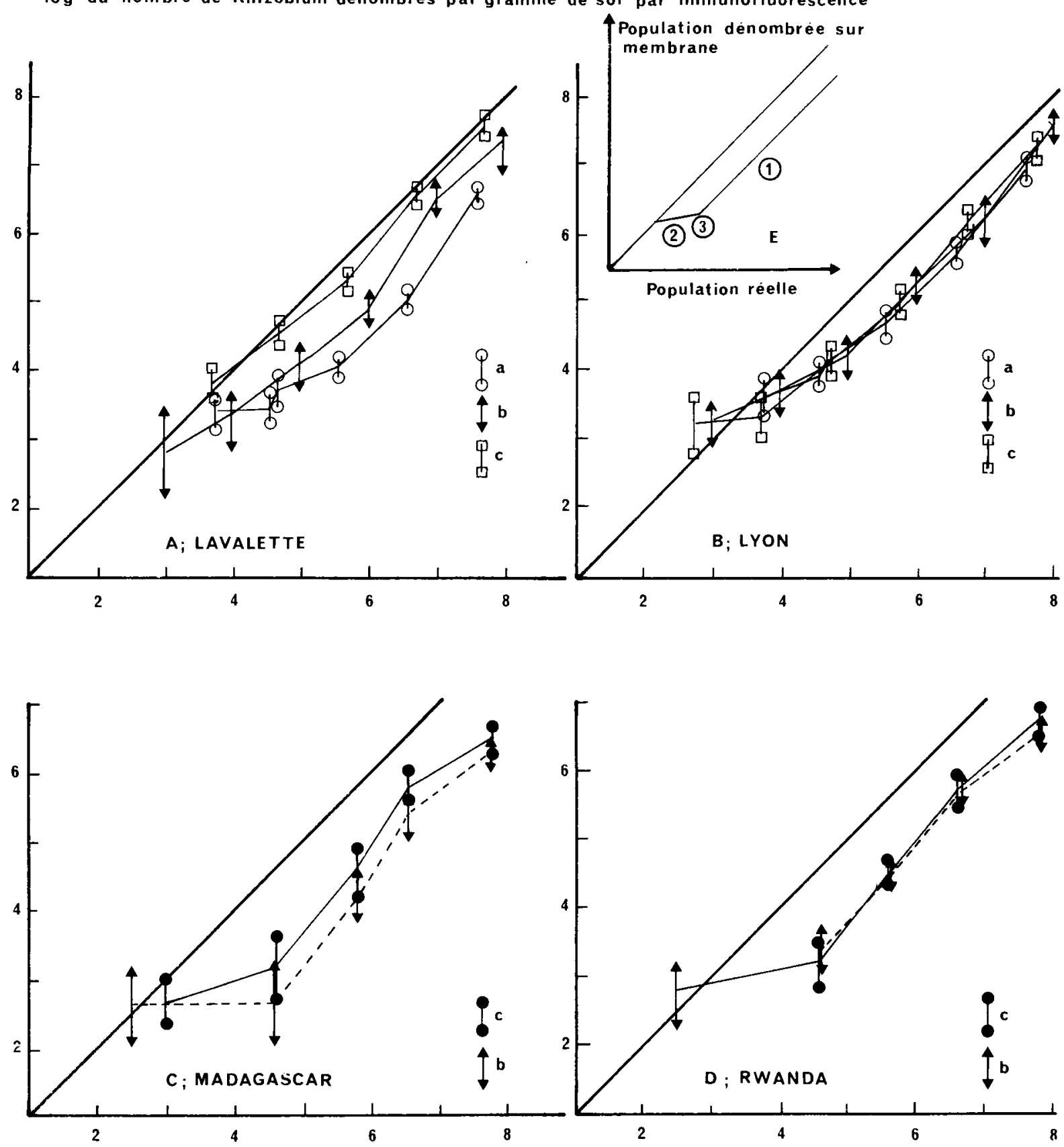

log du nombre de Rhizobium introduit dans le sol (dénombrement sur lames)

Figure 7A.B.C.D.E.

Cinétique de récupération de $\mathrm{R}$. japonicum (souche $G_{2} s p$ ) dans des sols de régions tempérées $(A:$ Lavalette $-B:$ Lyon) et de régions tropicales (C: Madagascar $-D: R$ wanda).

Comparaison des protocoles expérimentaux:

$A$ et $B:$ a) classique
b) sur polycarbonate
c) gélatine

$C$ et $D$ : b) polycarbonate

c) gélatine

E: Schéma d'une cinétique de récupération (1) Zone de proportionnalité, (2) Zone de latence (non-proportionnalité), (3) Zone de résolution ou de non-proportionnalité.
Kinetics of recovery of $\mathrm{R}$. japonicum (strain $G_{2} s p$ ) in temperate soils $(A:$ Lavalette $-B:$ Lyon) and in tropical soils $(C:$ Madagascar $-D: R$ wanda).

$A$ and $B:$ a) classical procedure

b) polycarbonate filters

c) gelatin

$C$ and $D: b)$ polycarbonate filters

c) gelatin

$E:$ Schematic diagram of a kinetic of recovery. 
Dans le cadre d'études de la dynamique des populations de micro-organismes telluriques, il importe de définir les limites d'utilisation de la méthode de dénombrement par immunofluorescence, ceci pour chaque sol et chaque nouvel organisme considéré. L'ensemble de ces résultats permet de définir une méthodologie adaptable à tout matériel :

1) Contrôles préliminaires concernant l'utilisation des anticorps marqués (CLEYET-MAREL, 1979).

2) Contrôle de la distribution des micro-organismes sur les membranes en polycarbonates et élaboration d'un protocole d'observation (choix d'une précision relative).

3) Choix d'un floculant et d'un temps de floculation: meilleur compromis entre une faible D.O. $(660 \mathrm{~nm})$ et un temps n'engendrant pas trop de pertes.

4) Choix de l'extractif autorisant la meilleure récupération. Détermination du seuil de résolution.

Reçu le 17 juin 1983. Accepté le 8 mars 1984.

\section{RÉFÉRENCES BIBLIOGRAPHIQUES}

Bezdicek D.F., Donaldson M. D., 1980. Flocculation of Rhizobium from soil colloids for enumeration by immunofluorescence, 297-309. In Berkeley R. C. W., Lynch J. M., Melling J. M., Rutter, P. R., Vincent B. : « Microbial adhesion to surfaces ». Ellis-Horwood ed., Chichester, $343 \mathrm{p}$.

Bohlool B. B., Schmidt E. L., 1980 : The immunofluorescent approach in microbial ecology, 203-241. In Alexander M. « Advances in Microbial ecology », Plenum Press ed., New York \& London, $247 \mathrm{p}$.

Cleyet-Marel J.-C., 1979. Apport de l'immunofluorescence à la connaissance de l'écologie de Rhizobium japonicum dans le sol et la rhizosphère. Thèse $3^{\mathrm{e}}$ cycle, Univ. Claude-Bernard, Lyon I, $122 \mathrm{p}$.

Cleyet-Marel J.-C., Chessel D., 1978. Méthode de dénombrement de Rhizobium japonicum par immunofluorescence. Analyse statistique des comptages. Ann. Phytopathol., 10 (2), 219-231.

Cleyet-Marel J.-C., Crozat Y., 1982. Etude écologique en immunofluorescence de Rhizobium japonicum dans le sol et la rhizosphère. Agronomie, 2, (3), 243-248.

Crozat Y., 1983. Caractérisation du pouvoir saprophyte des souches de Rhizobium japonicum dans le sol à l'aide de l'immunofluorescence. Thèse $3^{\mathrm{e}}$ cycle, Univ. Claude Bernard, Lyon I., $153 \mathrm{p}$.
Crozat Y., Cleyet-Marel J.-C., Giraud J. J., Obaton M., 1982. Survival rates of Rhizobium japonicum populations introduced into different soils. Soil Biol. Biochem., 14, 401-405.

Kingsley M. T., Bohlool B. B., 1981. Release of Rhizobium spp. from tropical soils and recovery for immunofluorescence enumeration. Appl. environm. Microbiol., 42, 241-248.

Reyes V. G., Schmidt E. L., 1979. Population densities of Rhizobium japonicum strain 123 estimated directly in soil and rhizosphere. Appl. environm. Microbiol., 37, 854-858.

Schmidt E. L., 1974. Quantitative autecological study of microorganisms in soils by immunofluorescence. Soil Sci., 118, 141-149.

Schmidt E. L., 1978. Ecology of the legume root nodule bacteria, 269-303. In Dommergues Y. R. \& Krupa S. V., «Interactions between non pathogenic soil microorganisms and plants ", Elsevier Publ., 475 p.

Vincent J. M., 1970. A manual for the practical study of rootnodule bacteria. Oxford, Edinburgh, Blackwell Sci. Publi., 164 p.

Wollum A. G., Miller R. H., 1980. A density centrifugation method for recovering Rhizobium spp. from soil for fluorescent antibody studies. Appl. Environm. Microbiol., 39, 466-469. 